\title{
Emission-line stars in the LMC: the Armagh survey and a metacatalogue ${ }^{\star}$ (Research Note)
}

\author{
I. D. Howarth
}

\author{
Dept. of Physics \& Astronomy, UCL, Gower St., London WC1E 6BT, UK \\ e-mail: idh@star.ucl.ac.uk
}

Received 7 May 2013 / Accepted 10 June 2013

\section{ABSTRACT}

\begin{abstract}
Aims. Accurate astrometry is required to reliably cross-match 20th-century photographic catalogues against 21 st-century digital surveys. The present work provides modern-era identifications and astrometry for the 801 emission-line objects "of stellar appearance" in the Armagh survey (the largest of its nature to date).

Methods. Targets have been individually identified in digital images using the Armagh Atlas and, in most cases, unambiguously matched to entries in the UCAC astrometric catalogues.

Results. Astrometry with sub-arcsecond precision is now available for all the major photographic spectroscopic surveys of the LMC. The results are used to compile an annotated metacatalogue of 1675 individual, spectroscopically identified candidate H $\alpha$-emission stars, including detailed cross-matching between catalogues, and resolving many (though not all) identification ambiguities in individual primary sources.
\end{abstract}

Key words. astrometry - stars: emission-line, Be - Magellanic Clouds - catalogs

\section{Introduction}

The Large Magellanic Cloud (LMC) provides an unsurpassed laboratory for the investigation of massive stars; its low reddening and relative proximity facilitate both large-scale surveys and detailed studies of individual objects of interest. As a consequence, a number of extensive photographic searches for luminous LMC stars with $\mathrm{H} \alpha$ emission were conducted as technological advances allowed, first by Henize (1956; 172 "LH $\alpha 120-\mathrm{S}$ " stars), and subsequently at Armagh (Lindsay 1963; Andrews \& Lindsay 1964; 801 "L63" and "AL" stars) and by Bohannan \& Epps (1974; 625 "BE74" stars), alongside more general surveys, such as Sanduleak's (Sanduleak 1970). At the time of writing, the Armagh and BE74 surveys still provide the two largest lists of spectroscopically identified candidate LMC emissionline stars available.

The utility of all these catalogues has been limited by coordinates that were originally given to only $\sim$ arcminute precision - inadequate for reliable identifications based on position alone. While accompanying, relatively small-scale finder charts normally provide a more secure route to identification, in the context of large-scale digital surveys the task of visually checking many targets against numerous published finding charts is discouragingly onerous, a problem common to all the early major surveys. The situation has gradually improved in recent years, particularly as Brian Skiff (Lowell Observatory) has obtained modern identifications, and hence precise astrometry, for the major photographic objective-prism surveys of LMC targets, as one aspect of his extensive and continuing on-line Catalogue of Stellar Spectral Classifications, commonly cited as "B/mk"

* Tables A1-A4 are only available at the CDS via anonymous ftp to cdsarc.u-strasbg.fr (130.79.128.5) or via

http://cdsarc.u-strasbg.fr/viz-bin/qcat? J/A+A/555/A141
(Skiff 2013). His efforts have provided precise positions for the Henize (1956) listing, among others, while Howarth (2012) has given identifications and astrometry for the BE74 catalogue.

The last (and largest) of the major emission-line catalogues in need of systematic, precise positions is the Armagh survey. This was published in three tranches; the first contains objects with no detectable continuum, interpreted as emission nebulae (Lindsay \& Mullan 1963). The second and third lists are of "objects of stellar appearance" (Lindsay 1963, L63; Andrews \& Lindsay 1964, AL); it is these stellar sources that are the principal subject of this Note.

\section{Methods}

Lindsay (1963) and Andrews \& Lindsay (1964) initially published only approximate co-ordinates, but, after Lindsay's death, finder charts were published at Armagh Observatory by McFarland (McFarland et al. 1975, hereinafter MLA), in the form of a folder of loose-leaf reproductions of blue (103a-0) plates at a scale of $\sim 16^{\prime \prime} / \mathrm{mm}$.

Using these charts, counterparts of the Armagh emissionline stars have been visually identified on red images of the second Digitized Sky Survey (DSS2), using the CDS's Aladin tool; where necessary, identifications were refined as described in Sect. 3. The corresponding positions were recorded interactively, and transferred to a data file by copy-and-paste. Correlating the results against UCAC4 (Zacharias et al. 2012) gave a positive match with a single target within $5^{\prime \prime}$ in most cases, with a subarcsecond systematic offset. After correcting the interactively recorded measurements for this offset, a second pass was made against UCAC4 with a $2^{\prime \prime}$-radius window to obtain final positions. Positional differences between corrected interactive measurements and UCAC4 are less than one arc-second in the great majority of cases. 
Table 1. Metacatalogue composition.

\begin{tabular}{lrrrrrrrr}
\hline \hline & LH $\alpha$ & L63 & AL & BE74 & BAT & RPs & First & Unique \\
\hline LH $\alpha$ 120-S & $\mathbf{1 7 2}$ & 53 & 72 & 136 & 16 & 23 & 172 & 14 \\
L63 & & $\mathbf{3 5 8}$ & 3 & 137 & 19 & 45 & 305 & 191 \\
AL & & & $\mathbf{4 4 6}$ & 172 & 31 & 34 & 372 & 241 \\
BE74 & & & & $\mathbf{6 2 4}$ & 53 & 74 & 284 & 242 \\
BAT99 & & & & 55 & $\mathbf{1 3 4}$ & 0 & 66 & 66 \\
RPs & 25 & & & & & $\mathbf{5 7 6}$ & 467 & 467 \\
\hline
\end{tabular}

Notes. Entries show the number of stars common to each pair of primary catalogues (e.g., there are 172 entries in the LH $\alpha$ 120-S listing, of which 53 are also in the L63 catalogue). Twenty-five RPs catalogue entries have LH $\alpha$ 120-S matches, but only 23 LH $\alpha$ 120-S entries have RPs entries, because LH $\alpha$ 120-S 24 corresponds to three separate RPs objects; similarly, BE74-383 (HD 269828) matches three separate BAT99 entries. The last two columns give the number of "discovery" entries (e.g., of the 446 entries in the AL catalogue, 372 had not been previously identified by Henize 1956 or by Lindsay 1963), and the number of entries not appearing in any of the other tabulated catalogues. The RPs study is targeted on fainter objects than the 20th-century surveys, giving rise to a large number of new targets, with relatively few recoveries of known, brighter stars. The BE74 total of 624 is one fewer than the number of their catalogue entries because BE74 394 is not marked on their charts, and the counterpart is not reliably identifiable. The RPs total of 576 stars is three fewer than their asserted 579 objects because their Table A.1 has only 578 entries, including one duplicated listing (RPs1822) and one duplicated target (RPs447 = RPs1014). The sum of "First" discoveries (1666) is nine fewer than the total number of metacatalogue entries (1675), because (i) LH $\alpha$ 120-S 24 and BE74-383 are two "firsts" that translate to six objects; and (ii) the metacatalogue includes five WR stars not included in the listed primary catalogues.

References. LH $\alpha$ 120-S = Henize (1956); L63 = Lindsay (1963); AL = Andrews \& Lindsay (1964); BE74 = Bohannan \& Epps (1974); BAT99 = Breysacher et al. (1999); RPs = Reid \& Parker (2012).

The results are listed in Table A.1 (on-line); cross-matches against the UCAC2 and 2MASS catalogues are included for the convenience of those who prefer these better-established sources (including consistency with $\mathrm{B} / \mathrm{mk}$ ), although the positional precision is now such that cross-matching against any catalogues of interest should be straightforward.

\section{Cross-identification metacatalogue}

The co-ordinates reported here are principally intended to establish precise positions for the objects marked by MLA on their finder charts. However, in a number of cases the charts present ambiguities or other uncertainties in identification ${ }^{1}$, and in an effort to alleviate these uncertainties and clarify correct identifications, the new Armagh results have been correlated with the Henize (1956) and Bohannan \& Epps (1974) lists, using the Skiff and Howarth positions, but referring back to the original finder charts as necessary.

The BAT99 catalogue of 134 LMC Wolf-Rayet stars (Breysacher et al. 1999) and the recent Reid \& Parker (2012) catalogue of $\mathrm{H} \alpha$ emission-line stars (576 RPs stars) were also used in this effort, with matching based on positional coincidence alone; for both lists, good astrometry is available from the original sources ${ }^{2}$. For completeness, the BAT99 listing was augmented by the five subsequent LMC WR discoveries announced up to the end of 2012 (Evans et al. 2011; Gvaramadze et al. 2012; Howarth \& Walborn 2012; Neugent et al. 2012) 3 $^{3}$, although no effort has been made to include individual stars from other sources (not least because of the ambiguity of what properly constitutes an 'emission-line' star in the present context).

\footnotetext{
1 One contributing factor is that the scale and image quality of the MLA charts mean that it is not feasible to resolve stars separated by less than $\sim 10^{\prime \prime}$, at best (cp. DSS2 imaging, which allows multiple objects to be identified at separations down to $\lesssim 3^{\prime \prime}$ ).

2 For convenience, the BAT99 source positions were actually taken from Bonanos et al. (2009), which has reliable, precise astrometry for all these targets (though their compilation of LMC spectral types is much less complete than $\mathrm{B} / \mathrm{mk}$ ).

3 In addition, Massey et al. (2000) newly identify Sk $-69^{\circ} 194$ (L63 289) as a WR star.
}

The resulting metacatalogue amalgamates all the major spectroscopically-based surveys dedicated to finding LMC emission-line candidates, and yields 1675 stars that (probably) represent separate objects. The metacatalogue is given in Table A.2, with comments in Table A.3 (both on-line), while Table 1 summarizes the contributions from the various primary sources. Since many targets are known under several names, a unique running "LMCe" number is assigned in Table A.2, for internal cross-referencing to other Tables (and not to promulgate yet another label for objects which already all have at least one unique identifier).

Cross-matches of the metacatalogue entries have been made with the Reid \& Parker planetary-nebula survey (Reid \& Parker 2006), and the Sabogal et al. (2005) catalogue of Be-star candidates (proposed on the grounds of characteristic photometric variability); this helped to resolve identification ambiguities in a few cases (particularly in crowded fields). For completeness, matches have also been made with the Henry Draper Extension (HDE; Cannon 1936), the Sanduleak catalogue (Sk; Sanduleak 1970), the Radcliffe luminous-star catalogue (RMC; Feast et al. 1960), and the VLT-Flames Tarantula Survey (VFTS; Evans et al. 2011). Results are included in Table A.2.

Duflot (2010) has compiled a related metacatalogue of LMC stars, of all types. His effort differs from that reported here both in its more ambitious scale (4011 entries), and in that he appears to rely on the co-ordinates published in primary sources as the main criterion for identifying catalogue matches (leaving the question of the actual stellar identifications unresolved in many cases, although Skiff has since provided modern identifications, and precise astrometry, for most of the targets). The current metacatalogue has been compared with Duflot's; results are included in Table A.2, and discrepancies discussed in Table A.3. In general, the agreement in matchings of primary catalogues is remarkably good; in only three cases does Duflot appear to assign physically different stars to a single identifier (see Table A.3 entries for LMCe 951, 1282, and 1285), while overlooking 10 matches identified here, and omitting two stars (AL-29 and BE74-527). Reassuringly (and surprisingly!), no revisions to the composition of the emission-line metacatalogue were required as a result of the comparison with Duflot (2010), 

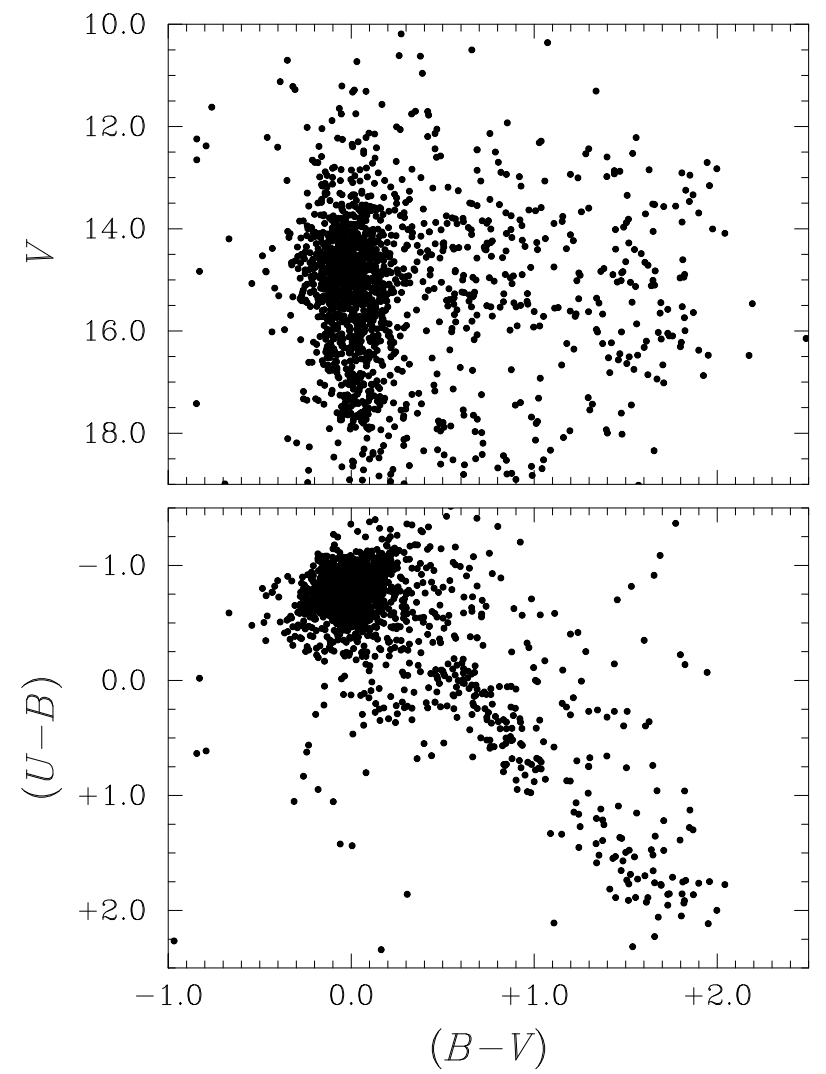

Fig. 1. Colour-magnitude and colour-colour diagrams for entries in the consolidated metacatalogue (MCPS photometry; Zaritsky et al. 2004).

encouraging a view that probably no more than a handful of straighforwardly identifiable errors remain.

\subsection{Overview of metacatalogue properties}

Figure 1 shows colour-magnitude and colour-colour diagrams for the metacatalogue stars. The photometry is taken from Zaritsky et al. (2004), to whom reference should be made for data-quality caveats. As expected, the samples are evidently dominated by luminous, blue stars, although a number of redder objects occur. While many of these redder objects may have intrinsic $\mathrm{H} \alpha$ emission, the objective-prism surveys may also include M-type stars in which the $\mathrm{TiO}$ bandhead at 654 nm "mocks" H $\alpha$ emission (MacConnell \& Coyne 1983; Skiff, priv. comm.).

UCAC4 proper motions are available for 1417 of the entries in Table A.2, with a median error of 3.6 mas $\mathrm{yr}^{-1}$. LMC members are not expected to show detectable proper motions at this level of accuracy; in practice, 264 stars have proper motions that are statistically formally significant at the 5- $\sigma$ level or above,

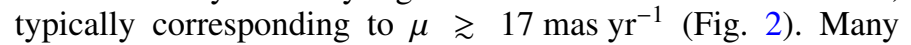
of these will be foreground objects, although some spuriously high proper motions must arise through errors. UCAC4 propermotion information is included in Table A.2, for guidance.

\section{Discussion}

The newly available redundancy among primary catalogues was often crucial in determining the correct identification of an emission-line source (or, at the least, in clarifying where ambiguities remain), as elaborated on a case by case basis in Table A.3. Consequently, 27 (4.3\%) of the BE74 identifications adopted by
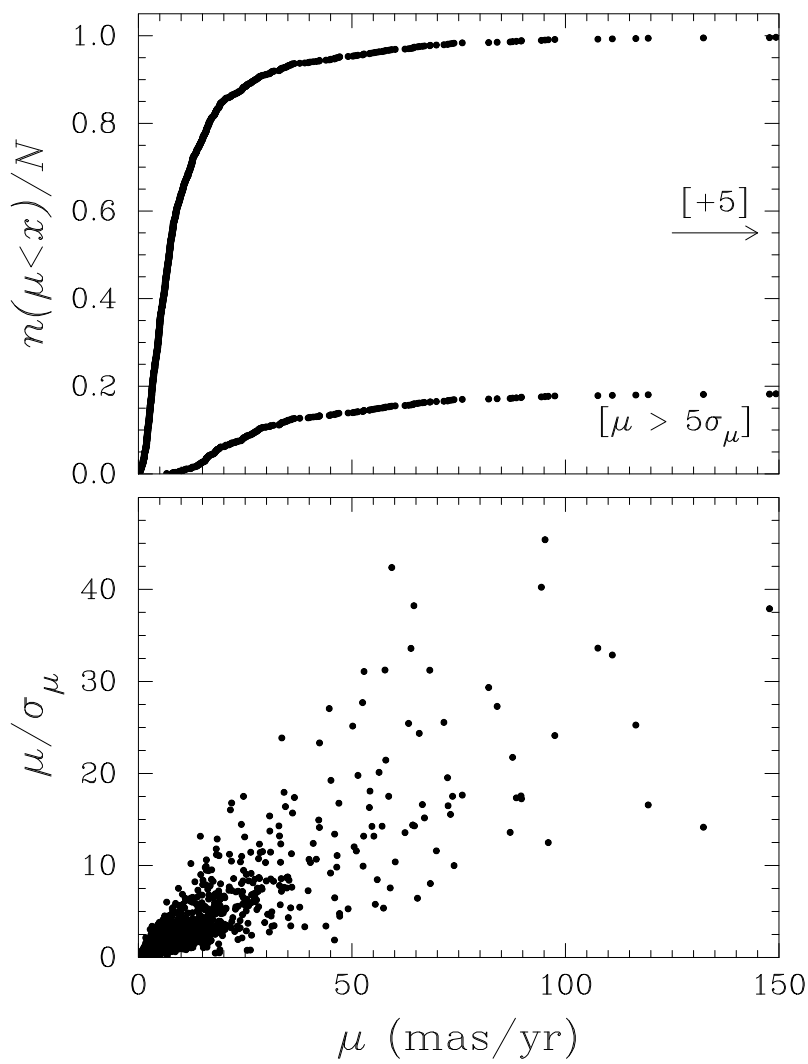

Fig. 2. Upper panel: cumulative distribution function of UCAC4 proper motions for 1417 stars in the consolidated metacatalogue, including the subset of 264 objects where the proper motion is more than $5 \times$ its error. Lower panel: detection significance.

Howarth (2012) are revised, as summarized in Table A.4 (online). Two of these revisions arise from gross errors (wrong star originally recorded), 13 from new or revised resolutions of ambiguities in the BE74 charts, and 12 from newly revealed probable errors by BE74.

The relatively bright Wolf-Rayet star BAT99 131 offers an interesting, and cautionary, illustration. Westerlund \& Smith (1964) discovered it under the name WS 51, and identified it (proleptically) with UCAC4 115-010182. Bohannan \& Epps (1974) independently recovered it under the guise of BE74 151, marking the same star on their charts as did Westerlund \& Smith (1964). Meanwhile, Sanduleak (1970) had marked his $\mathrm{Sk}-67^{\circ} 259$ as a star of similar brightness lying $13^{\prime \prime}$ to the SW (UCAC4 115-010180), identifying it with the WR; but Fehrenbach et al. (1976) later asserted that Sanduleak's identification was in error. Breysacher (1981) obtained new slit spectroscopy of the WR, as Brey 98, and implicitly confirmed the identification with the NE star (repeating Fehrenbach et al.'s assertion, although identifying Brey 98 with, inter alia, both Sk $-67^{\circ} 259$ and WS 51).

However, McFarland et al. (1975) identify AL 412 with the SW star (i.e., with Sk $-67^{\circ}$ 259), and Breysacher et al. (1999) give a finder chart, and precise astrometry, also identifying this as the WR, without further comment. Foellmi et al. (2003) and Crowther \& Hadfield (2006) observed the star; the former reproduce the BAT99 co-ordinates (again without comment), and Crowther (priv. comm.) confirms, on the basis of his finder charts and telescope pointing, that the SW component is a WR star. Either both stars are emission-line objects (both are blue in $B-V)$, but each investigation found only one; or, more plausibly, 
both Westerlund \& Smith (1964) and Bohannan \& Epps (1974) are in error, as is the "correction" in Fehrenbach et al. (1976).

No doubt similar cases remain to be resolved (several potential instances are discussed in Table A.3) but, as in this example, new spectroscopy is likely to be the only secure method of addressing these issues in general.

Acknowledgements. The HDE, RMC, LH $\alpha$ 120-S, and Sk co-ordinates used here are all Brian Skiff's work. I thank him for helpful correspondence, and not least for bringing the MLA charts to my attention. John McFarland \& Mark Bailey, of Armagh Observatory, kindly provided both a set of those charts, and a pdf of a high-quality scan (subsequently made more generally available by them, through the ADS). The referee, François Ochsenbein, suggested the comparison with the Duflot compilation. The execution of this work relied on resources and tools provided by the Centre de Données astronomiques de Strasbourg.

\section{References}

Andrews, A. D., \& Lindsay, E. M. 1964, Irish AJ, 6, 241

Bohannan, B., \& Epps, H. W. 1974, A\&AS, 18, 47

Bonanos, A. Z., Massa, D. L., Sewilo, M., et al. 2009, AJ, 138, 1003

Breysacher, J. 1981, A\&AS, 43, 203

Breysacher, J., Azzopardi, M., \& Testor, G. 1999, A\&AS, 137, 117

Cannon, A. J. 1936, Annals of Harvard College Observatory, 100, 208

Crowther, P. A., \& Hadfield, L. J. 2006, A\&A, 449, 711

Duflot, M. 2010, VizieR Online Data Catalog: V/131

Evans, C. J., Taylor, W. D., Hénault-Brunet, V., et al. 2011, A\&A, 530, A108
Feast, M. W., Thackeray, A. D., \& Wesselink, A. J. 1960, MNRAS, 121, 337

Fehrenbach, C., Duflot, M., \& Acker, A. 1976, A\&AS, 24, 379

Foellmi, C., Moffat, A. F. J., \& Guerrero, M. A. 2003, MNRAS, 338, 1025

Gvaramadze, V. V., Chené, A.-N., Kniazev, A. Y., \& Schnurr, O. 2012, in Four Decades of Research on Massive Stars, eds. L. Drissen, C. Robert, N. St-Louis, \& A. F. J. Moffat, ASP Conf. Ser., 465, 511

Henize, K. G. 1956, ApJS, 2, 315

Howarth, I. D. 2012, A\&A, 548, A16

Howarth, I. D., \& Walborn, N. R. 2012, MNRAS, 426, 1867

Lindsay, E. M. 1963, Irish AJ, 6, 127

Lindsay, E. M., \& Mullan, D. J. 1963, Irish AJ, 6, 51

MacConnell, D. J., \& Coyne, G. V. 1983, Vatican Observatory Publications, 2, 63

Massey, P., Waterhouse, E., \& DeGioia-Eastwood, K. 2000, AJ, 119, 2214

McFarland, J., Lindsay, E. M., \& Andrews, A. D. 1975, Atlas for the Armagh survey of H-alpha emission objects in the Large Magellanic Cloud (Armagh: Armagh Observatory)

Neugent, K. F., Massey, P., \& Morrell, N. 2012, AJ, 144, 162

Reid, W. A., \& Parker, Q. A. 2006, MNRAS, 373, 521

Reid, W. A., \& Parker, Q. A. 2012, MNRAS, 425, 355

Sabogal, B. E., Mennickent, R. E., Pietrzyński, G., \& Gieren, W. 2005, MNRAS, 361, 1055

Sanduleak, N. 1970, Contributions from the Cerro Tololo Inter-American Observatory, 89

Skiff, B. A. 2013, VizieR Online Data Catalog, II/023

Westerlund, B. E., \& Smith, L. F. 1964, MNRAS, 128, 311

Zacharias, N., Finch, C. T., Girard, T. M., et al. 2012, The fourth US Naval Observatory CCD Astrograph Catalog (UCAC4); VizieR Online Data Catalog, II/322

Zaritsky, D., Harris, J., Thompson, I. B., \& Grebel, E. K. 2004, AJ, 128, 1606 